\title{
Hyperbolic Limit \\ of Parabolic Semilinear Heat Equations with Fading Memory
}

\author{
V. Pata
}

\begin{abstract}
This paper is devoted to the comparison of two models describing heat conduction with memory, arising in the frameworks of Coleman-Gurtin and Gurtin-Pipkin. In particular, the second model entails an equation of hyperbolic type, where the dissipation is carried out by the memory term solely, and can be viewed as the limit of the first model as the coefficient $\omega$ of the laplacian of the temperature tends to zero. Results concerning the asymptotic behavior, with emphasis on the existence of a uniform attractor, are provided, uniformly in $\omega$. The attractor of the hyperbolic model is shown to be upper semicontinuous with respect to the family of attractors of the parabolic models, as $\omega$ tends to zero.

Keywords: Heat equation, materials with memory, non-autonomous dynamical systems, uniform absorbing sets, uniform attractors, Hausdorff semidistance, upper semicontinuity of a family of attractors
\end{abstract}

AMS subject classification: 35B40, 35G25, 45K05, 80A22

\section{Introduction}

Let $\Omega \subset \mathbb{R}^{3}$ be a fixed bounded domain occupied by a solid heat conductor. Setting for simplicity the specific heat of the conductor equal to 1 , and assuming only small variations of the absolute temperature and temperature gradient from equilibrium reference values, the internal energy $e: \Omega \times \mathbb{R} \rightarrow \mathbb{R}$ and the heat flux vector $\mathbf{q}: \Omega \times \mathbb{R} \rightarrow \mathbb{R}^{3}$ are described by the constitutive equations

$$
\begin{aligned}
e(x, t) & =e_{0}+\vartheta(x, t) \\
\mathbf{q}(x, t) & =-\omega \nabla \vartheta(x, t)-\int_{0}^{\infty} k(s) \nabla \vartheta(x, t-s) d s
\end{aligned}
$$

where $\vartheta: \Omega \times \mathbb{R} \rightarrow \mathbb{R}$ is the temperature variation field relative to the equilibrium reference value, $k: \mathbb{R}^{+} \rightarrow \mathbb{R}$ is the heat flux memory kernel, and the constants $e_{0}$ and $\omega$ denote the internal energy at equilibrium and the instantaneous conductivity, respectively. The above equations are derived in the theory of heat flow with memory, due to Coleman and Gurtin [7] (case $\omega>0$ ), and to Gurtin and Pipkin [17] (case $\omega=0)$.

V. Pata: Politecnico di Milano, Dip. di Mate. "F. Brioschi", I-20133 Milano, Italy pata@mate.polimi.it

ISSN 0232-2064 / \$2.50 C Heldermann Verlag Berlin 
When a nonlinear temperature dependent heat source $g(x, \vartheta(x, t))+f(t, x)$ is involved, the energy balance equation

$$
e_{t}+\nabla \cdot \mathbf{q}=g+f
$$

leads to the equation

$$
\vartheta_{t}(t)=\omega \Delta \vartheta(t)+\int_{0}^{\infty} k(s) \Delta \vartheta(t-s) d s+g(\cdot, \vartheta(t))+f(t) .
$$

We assume that $\vartheta$ satisfies a Dirichlet boundary condition on $\partial \Omega$. Furthermore, the value of $\vartheta(t, x)$ is known for $t \leq \tau$, where $\tau \in \mathbb{R}$ is the initial time.

Our purpose is to perform a global asymptotic analysis of (1.1). In order to achieve this goal, we first have to associate with (1.1) a strongly continuous process of operators, which carries the initial data given at time $\tau$ to the solution at time $t$. A way to do that is to introduce the (integrated) past history of the temperature as a new variable of the problem. Hence, along the lines of $[9,13,15]$, we define

$$
\eta^{t}(x, s)=\int_{t-s}^{t} \vartheta(x, \sigma) d \sigma \quad\left(s \in \mathbb{R}^{+}\right) .
$$

Assuming $k(\infty)=0$ and setting $\mu=-k^{\prime}$, making a formal integration by parts we are led to the following initial and boundary value problem, depending on $\omega \geq 0$ :

Problem $\mathbf{P}_{\omega}$. Find $(\vartheta, \eta)$ solution to the system

$$
\begin{aligned}
& \vartheta_{t}(t)=\omega \Delta \vartheta(t)+\int_{0}^{\infty} \mu(s) \Delta \eta^{t}(s) d s+g(\cdot, \vartheta(t))+f(t) \\
& \eta_{t}^{t}(s)+\eta_{s}^{t}(s)=\vartheta(t)
\end{aligned}
$$

in $\Omega$, for any $t>\tau$ and any $s>0$, which satisfies the initial and boundary conditions

$$
\begin{aligned}
\omega \vartheta(t) & =0 & & \text { on } \partial \Omega \times(\tau,+\infty) \\
\int_{0}^{\infty} \mu(s) \eta^{t}(s) d s & =0 & & \text { on } \partial \Omega \times(\tau,+\infty) \\
\eta^{t}(0) & =0 & & \text { in } \Omega \times(\tau,+\infty) \\
\vartheta(\tau) & =\vartheta_{0} & & \text { in } \Omega \\
\eta^{\tau} & =\eta_{0} & & \text { in } \Omega \times \mathbb{R}^{+} .
\end{aligned}
$$

We point out that problem $\mathbf{P}_{\omega}$ is in fact equivalent to the original equation (1.1). Indeed, up to chosing

$$
\eta_{0}(x, s)=\int_{\tau-s}^{\tau} \vartheta(x, \sigma) d \sigma \quad\left(s \in \mathbb{R}^{+}\right)
$$

the first component $\vartheta$ of the solution $(\vartheta, \eta)$ to problem $\mathbf{P}_{\omega}$ is a solution to equation (1.1).

The presence of the parameter $\omega$ is substantial. When $\omega \neq 0$, the equation for $\vartheta$ is of parabolic type. In particular, the term $\omega \Delta \vartheta$, besides having a regularizing effect, 
gives a non-trivial contribution to the dissipation of the system. This setting has been analyzed in detail in the work [13], where it is shown that such a system possesses a uniform attractor for solutions. Conversely, the hyperbolic case $\omega=0$ is much more delicate. Indeed, in this situation all the dissipation of the system is contained in the convolution term. In the paper [15], there is an analysis of the linear case: by means of semigroup techniques, it is shown that the semigroup associated to the linear homogeneous system has an exponential decay, provided reasonable hypotheses on the kernel are assumed. However, the semigroup approach is not suitable to get information about the longtime behavior of solutions in presence of a non-linearity.

On the contrary, in this work, we pursue a different strategy. Namely, we try to get uniform estimates in time, using an argument which has been developed in [14]. This allows us give a quite accurate description of the asymptotic properties of the solutions to problem $\mathbf{P}_{0}$ for a certain class of non-linearities. Furthermore, with additional restrictions on the nonlinear term, it is possible to demonstrate that the system exhibits a uniform attractor. The next goal is a comparison between the parabolic and the hyperbolic cases, which shows an asymptotic continuity property of the models in the limit $\omega \rightarrow 0$. The key step in such analysis is the existence of estimates for the solutions which are independent of $\omega$. A similar argument has also been used in [16].

The plan of the paper is as follows. After some notation in Section 2, we prove existence and uniqueness results in Section 3 on any time interval $[\tau, T]$ for an external source $f \in L^{2}\left([\tau, T], L^{2}\right)$, under proper assumptions on the nonlinear term and on the memory kernel. In particular, we express the solution in terms of a strongly continuous process $U_{f}^{\omega}(t, \tau)$. In Section 4, under more restrictive hypotheses on $g$ and $\mu$, we show the existence of an absorbing set for $U_{f}^{\omega}(t, \tau)$, which is uniform as $f$ belongs to a certain functional space. Section 5 is devoted to the existence of a uniform attractor. Further restrictions on $f$ are then needed, namely, $f$ will belong to the hull of a certain translation compact function. Finally, in Section 6 we study the hyperbolic limit $\omega \rightarrow 0$. For simplicity, here we consider $f$ constant in time. In particular, being now the system autonomous, $S_{\omega}(t):=U_{f}^{\omega}(t, 0)$ turns out to be a strongly continuous semigroup.

\section{Notation}

Let $\Omega \subset \mathbb{R}^{3}$ be a bounded domain with smooth boundary $\partial \Omega$. With standard notation, we introduce the Hilbert spaces $H^{-1}, L^{2}, H_{0}^{1}, H^{2}$ on $\Omega$. We shall denote by $\langle\cdot, \cdot\rangle$ and $\|\cdot\|$ the inner product and the norm on $L^{2}$, respectively. The symbol $\langle\cdot, \cdot\rangle$ will also indicate the duality map between $H^{-1}$ and $H_{0}^{1}$. Identifying $L^{2}$ with its dual space, we have the compact and dense embeddings

$$
H^{-1} \hookrightarrow L^{2} \hookrightarrow H_{0}^{1} \hookrightarrow H^{2} \cap H_{0}^{1}
$$

Recalling the Poincaré inequality

$$
\|u\|^{2} \leq \lambda_{0}\|\nabla u\|^{2} \quad\left(u \in H_{0}^{1}\right)
$$

where $\lambda_{0}>0$ is the first eigenvalue of $-\Delta$ (with Dirichlet boundary conditions), the inner products in $H_{0}^{1}$ and $H^{2} \cap H_{0}^{1}$ can be chosen to be $\langle\nabla \cdot \nabla \cdot\rangle$ and $\langle\Delta \cdot, \Delta \cdot\rangle$, respectively. 
Given a positive function $\alpha$ defined on $\mathbb{R}^{+}$and a real Hilbert space $X$, let $L_{\alpha}^{2}\left(\mathbb{R}^{+}, X\right)$ be the Hilbert space of $X$-valued functions on $\mathbb{R}^{+}$endowed with the inner product

$$
\left\langle\psi_{1}, \psi_{2}\right\rangle_{L_{\alpha}^{2}\left(\mathbb{R}^{+}, X\right)}=\int_{0}^{\infty} \alpha(s)\left\langle\psi_{1}(s), \psi_{2}(s)\right\rangle_{X} d s
$$

Accordingly, the Hilbert space $H_{\alpha}^{1}\left(\mathbb{R}^{+}, X\right)$ is defined by

$$
H_{\alpha}^{1}\left(\mathbb{R}^{+}, X\right)=\left\{\psi(s): \mathbb{R}^{+} \rightarrow X \mid \psi, \psi_{s} \in L_{\alpha}^{2}\left(\mathbb{R}^{+}, X\right)\right\}
$$

Finally, we introduce the Banach space $\mathcal{T}(X)$ of $L_{\text {loc }}^{1}(\mathbb{R}, X)$-translation bounded functions with values in $X$, namely,

$$
\mathcal{T}(X)=\left\{h \in L_{\text {loc }}^{1}(\mathbb{R}, X):\|h\|_{\mathcal{T}(X)}=\sup _{r \in \mathbb{R}} \int_{r}^{r+1}\|h(y)\|_{X} d y<\infty\right\}
$$

A function $h \in \mathcal{T}(X)$ is said to be translation compact in $L_{\text {loc }}^{1}(\mathbb{R}, X)$ (see [6] and references therein) if the hull of $h$ defined as

$$
\mathrm{H}(h)=\overline{\left\{h^{r}\right\}_{r \in \mathbb{R}}} L_{\text {loc }}^{1}(\mathbb{R}, X)
$$

is compact in $L_{\text {loc }}^{1}(\mathbb{R}, X)$, where $h^{r}(\cdot)=h(\cdot+r)$ is the translate of $h$ by $r$. Hence a function $\varphi: \mathbb{R} \rightarrow X$ belongs to $\mathrm{H}(h)$ if and only if for any $\varepsilon>0$ and any $M>0$ there is a translate $h^{r}$ of $h$ such that $\int_{-M}^{M}\left\|\varphi(y)-h^{r}(y)\right\|_{X} d y \leq \varepsilon$. In particular, the relation $\|\varphi\|_{\mathcal{T}(X)} \leq\|h\|_{\mathcal{T}(X)}$ holds for every $\varphi \in \mathrm{H}(h)$. If $h$ is constant, then $\mathrm{H}(h)$ reduces to the singleton $\{h\}$. The class of translation compact functions in $L_{\text {loc }}^{1}(\mathbb{R}, X)$ is quite general; for instance, it contains $L^{p}(\mathbb{R}, X)$ for all $p \geq 1$, the constant $X$-valued functions, and the class of almost periodic functions (see [1]).

\section{The solutions process}

The first step is to write problem $\mathbf{P}_{\omega}$ in a proper functional setting. To this aim, we list some assumptions on the memory kernel $\mu$ and on the non-linearity $g$, which will be needed in the sequel.

Conditions on $\mu$. We consider the following hypotheses:

(h1) $\mu \in C^{1}\left(\mathbb{R}^{+}\right) \cap L^{1}\left(\mathbb{R}^{+}\right)$.

(h2) $\mu(s) \geq 0$ and $\mu^{\prime}(s) \leq 0$ for all $s \in \mathbb{R}^{+}$.

(h3) $\int_{0}^{\infty} \mu(s) d s=k_{0}>0$.

(h4) $\mu^{\prime}(s)+\delta \mu(s) \leq 0$ for all $s \in \mathbb{R}^{+}$and some $\delta>0$.

(h5) There exists $s_{0} \in \mathbb{R}^{+}$such that

- $\mu^{\prime} \in L^{2}\left(\left(0, s_{0}\right)\right)$

- $\mu^{\prime}(s)+M \mu(s) \geq 0$ for all $s \geq s_{0}$ and some $M>0$. 
It is clear that we can weaken the first of the two conditions in (h5) asking that $\mu^{\prime}$ is square summable in a neighborhood of zero. Then, by (h1), $\mu^{\prime} \in L^{2}\left(\left(0, s_{0}\right)\right)$ for every $s_{0} \in \mathbb{R}^{+}$. Also, notice that if the second condition of (h5) holds for every $s \in \mathbb{R}^{+}$, this automatically implies that $\mu^{\prime} \in L^{2}\left(\left(0, s_{0}\right)\right)$ for every $s_{0} \in \mathbb{R}^{+}$.

The above assumptions are in some sense quite restrictive. For instance, (h4) - (h5), which are not needed to prove existence and uniqueness results, imply the exponential decay of $\mu(s)$ and the finiteness of $\mu(0)$. However, all the results we found in literature concerning the exponential decay of linear semigroups, with dissipation contained in the sole memory term, are obtained requiring the exponential decay of the kernel. In our case, we do have some further dissipation when $\omega \neq 0$. But since we want to consider the behavior of the system in the limit $\omega \rightarrow 0$, we also have to require that $\mu \neq \equiv 0$, and this explains (h3).

Conditions on $g$. We consider the following hypotheses: let $g \in C^{0}(\Omega \times \mathbb{R})$, and assume that there exist non-negative constants $c_{1}, c_{2}$ such that

(g1) $|g(x, r)| \leq c_{1}(1+|r|)$ for all $x \in \Omega$ and all $r \in \mathbb{R}$.

(g2) $|g(x, r)-g(x, s)| \leq c_{2}|r-s|$ for all $x \in \Omega$ and all $r, s \in \mathbb{R}$.

(g3) $\limsup _{r \rightarrow \infty} \frac{g(x, r)}{r} \leq 0$ uniformly as $x \in \Omega$.

(g4) $g(x, \cdot) \in C^{1}(\Omega \times \mathbb{R})$ and

- $g(\cdot, 0) \in H_{0}^{1}$

- $g^{\prime}(\cdot, r) \leq 0$ for all $r \in \mathbb{R}$.

- $\sup _{\|u\| \leq c}\left\|D_{x} g(\cdot, u)\right\|_{L^{2}}<\infty$ for all $c \geq 0$.

Here $D_{x}$ and the prime denote derivation with respect to the first three space variables, and derivation with respect to the fourth variable of $g$, respectively. Notice that (g4) together with (g1) imply (g3). Conditions (g1) - (g2) will be enough to assure existence and uniqueness; (g3) will be used to prove the existence of a uniform absorbing set, whereas (g4) is needed for the uniform attractor.

Again, the above conditions are too restrictive in the parabolic case $\omega>0$ (see [13]). On the other hand, in the hyperbolic situation, that is, when $\omega=0$, even a linear $g(x, r)=\varepsilon r$, with $\varepsilon>0$ very small, could prevent the system from being dissipative. Notice that, if we consider the linear homogeneous case corresponding to such $g$, it is easy to check that the the strongly continuous semigroup of linear operators associated to the equation is not a contraction semigroup (cf. [15]).

Definition 3.1. Assume (h1) - (h2) and (g1). Let $\tau, T \in \mathbb{R}, T>\tau$, and set $I=[\tau, T]$. Assume also that

$$
f \in L^{1}\left(I, L^{2}\right) .
$$

A pair $(\vartheta, \eta)$ which fulfils

$$
\begin{aligned}
\vartheta & \in C^{0}\left(I, L^{2}\right) \\
\omega \vartheta & \in L^{2}\left(I, H_{0}^{1}\right) \\
\vartheta_{t} & \in L^{\infty}\left(I, H^{-1}\right)+L^{1}\left(I, L^{2}\right) \\
\eta & \in C^{0}\left(I, L_{\mu}^{2}\left(\mathbb{R}^{+}, H_{0}^{1}\right)\right) \\
\eta_{t}+\eta_{s} & \in C^{0}\left(I, L_{\mu}^{2}\left(\mathbb{R}^{+}, L^{2}\right)\right)
\end{aligned}
$$


is a solution to problem $\mathbf{P}_{\omega}$ in the time interval $I$ provided that

$$
\begin{aligned}
& \left\langle\vartheta_{t}, v\right\rangle=-\omega\langle\nabla \vartheta, \nabla v\rangle-\int_{0}^{\infty} \mu(s)\langle\nabla \eta(s), \nabla v\rangle d s+\langle g(\cdot, \vartheta), v\rangle+\langle f, v\rangle \\
& \int_{0}^{\infty} \mu(s)\left\langle\eta_{t}(s)+\eta_{s}(s), \Delta \zeta(s)\right\rangle d s=\int_{0}^{\infty} \mu(s)\langle\vartheta, \Delta \zeta(s)\rangle d s
\end{aligned}
$$

for every $v \in H_{0}^{1}$ and every $\zeta \in L_{\mu}^{2}\left(\mathbb{R}^{+}, H^{2} \cap H_{0}^{1}\right)$, a.e. in $I$, with initial conditions

$$
\begin{array}{rlrl}
\vartheta(\tau) & =\vartheta_{0} & & \text { a.e. in } \Omega \\
\eta^{\tau}=\eta_{0} & & \text { a.e. in } \Omega \times \mathbb{R}^{+} .
\end{array}
$$

Here $\partial_{s}$ is a linear operator on $L_{\mu}^{2}\left(\mathbb{R}^{+}, H_{0}^{1}\right)$ of domain

$$
\mathcal{D}\left(\partial_{s}\right)=\left\{\psi \in H_{\mu}^{1}\left(\mathbb{R}^{+}, H_{0}^{1}\right) \mid \psi(0)=0\right\} .
$$

The following result holds (cf. $[12,13,15]$ ).

Theorem 3.2. Let (h1) - (h2), (g1) - (g2), and (3.1) hold. Then, given any $\tau \in \mathbb{R}$ and any $T>\tau$, problem $\mathbf{P}_{\omega}$ has a unique solution $(\vartheta, \eta)$ in the time interval $I=[\tau, T]$, with initial data $\left(\vartheta_{0}, \eta_{0}\right)$. In addition, if we consider two sets of data $\left\{f_{i}, \vartheta_{0 i}, \eta_{0 i}\right\} \quad(i=$ $1,2)$ and we denote by $\left\{\vartheta_{i}, \eta_{i}\right\}$ the two corresponding solutions to problem $\mathbf{P}_{\omega}$, then there holds

$$
\begin{aligned}
\| \vartheta_{1}(t) & -\vartheta_{2}(t)\left\|^{2}+\int_{0}^{\infty} \mu(s)\right\| \nabla \eta_{1}^{t}(s)-\nabla \eta_{2}^{t}(s) \|^{2} d s \\
\leq & C\left[\left\|\vartheta_{01}-\vartheta_{02}\right\|^{2}+\int_{0}^{\infty} \mu(s)\left\|\nabla \eta_{01}(s)-\nabla \eta_{02}(s)\right\|^{2} d s\right. \\
& \left.+\left(\int_{\tau}^{T}\left\|f_{1}(y)-f_{2}(y)\right\| d y\right)^{2}\right] \quad(t \in I)
\end{aligned}
$$

for some constant $C>0$, depending (increasingly) only on the size of the data, but independent of $\omega \geq 0$.

Proof. The case $\omega>0$ is proved in [13]. Existence and uniqueness for the case $\omega=0$ can be recovered from [15] using a standard fixed point argument. Existence could also be proved via a Faedo-Galerkin scheme, or taking the limit as $\omega \rightarrow 0$ of the parabolic problem. Thus the only thing to be proved is continuous dependence, that is, estimate (3.2). Let then $\left\{\vartheta_{i}, \eta_{i}\right\} \quad(i=1,2)$ be two solutions to problem $\mathbf{P}_{0}$ corresponding to the source terms and initial data $\left\{f_{i}, \vartheta_{0 i}, \eta_{0 i}\right\}$, and denote their differences by $\{\vartheta, \eta\}$ and $\left\{f, \vartheta_{0}, \eta_{0}\right\}$, respectively. According to Definition 3.1 , the pair $(\vartheta, \eta)$ fulfils the system

$$
\begin{aligned}
& \left\langle\vartheta_{t}, v\right\rangle=-\int_{0}^{\infty} \mu(s)\langle\nabla \eta(s), \nabla v\rangle d s+\left\langle g\left(\cdot, \vartheta_{1}\right)-g\left(\cdot, \vartheta_{2}\right), v\right\rangle+\langle f, v\rangle \\
& \int_{0}^{\infty} \mu(s)\left\langle\eta_{t}(s)+\eta_{s}(s), \Delta \zeta(s)\right\rangle d s=\int_{0}^{\infty} \mu(s)\langle\vartheta, \Delta \zeta(s)\rangle d s \\
& \vartheta(\tau)=\vartheta_{0} \\
& \eta^{\tau}=\eta_{0}
\end{aligned}
$$


for every $v \in H_{0}^{1}$ and every $\zeta \in L_{\mu}^{2}\left(\mathbb{R}^{+}, H^{2} \cap H_{0}^{1}\right)$, a.e. in $I$. Since we already know that we have uniqueness, we can perform formal estimates in an approximation scheme, and then pass to the limit. Alternatively, one should work with regularized equations. A similar situation has been treated in detail in [10]. Setting $v=\vartheta$ in (3.3), and using (g2), we have

$$
\begin{aligned}
\frac{1}{2} \frac{d}{d t}\|\vartheta\|^{2} & =-\int_{0}^{\infty} \mu(s)\langle\nabla \eta(s), \nabla \vartheta\rangle d s+\left\langle g\left(\cdot, \vartheta_{1}\right)-g\left(\cdot, \vartheta_{2}\right), \vartheta\right\rangle+\langle f, \vartheta\rangle \\
& \leq-\int_{0}^{\infty} \mu(s)\langle\nabla \eta(s), \nabla \vartheta\rangle d s+c_{2}\|\vartheta\|^{2}\|f\|\|\vartheta\| .
\end{aligned}
$$

Setting $\zeta=\eta$ in (3.4), and exploiting (h2) and integration by parts (see [12] for the details), we end up with

$$
\begin{aligned}
\frac{1}{2} \frac{d}{d t} & \int_{0}^{\infty} \mu(s)\|\nabla \eta(s)\|^{2} d s \\
& =-\int_{0}^{\infty} \mu(s)\left\langle\nabla \eta_{s}(s), \nabla \eta(s)\right\rangle d s+\int_{0}^{\infty} \mu(s)\langle\nabla \eta(s), \nabla \vartheta\rangle d s \\
& =\frac{1}{2} \int_{0}^{\infty} \mu^{\prime}(s)\|\nabla \eta(s)\|^{2} d s+\int_{0}^{\infty} \mu(s)\langle\nabla \eta(s), \nabla \vartheta\rangle d s \\
& \leq \int_{0}^{\infty} \mu(s)\langle\nabla \eta(s), \nabla \vartheta\rangle d s .
\end{aligned}
$$

Addition of (3.7) - (3.8) leads to

$$
\frac{1}{2} \frac{d}{d t}\left(\|\vartheta\|^{2}+\int_{0}^{\infty} \mu(s)\|\nabla \eta(s)\|^{2} d s\right) \leq c_{2}\|\vartheta\|^{2}+\|f\|\|\vartheta\| .
$$

The thesis then follows from (3.5) - (3.6) and a Gronwall-type Lemma (see, e.g., [3: Lemma A.5] and [21: Appendix])

Introducing the product space

$$
\mathcal{H}=L^{2} \times L_{\mu}^{2}\left(\mathbb{R}^{+}, H_{0}^{1}\right),
$$

in light of Theorem 3.2, it is possible to express the solutions to problem $\mathbf{P}_{\omega}$ in terms of a strongly continuous process of operators (see [20]). Namely, we write the solution $\left(\vartheta(t), \eta^{t}\right)$ of problem $\mathbf{P}_{\omega}$ at time $t$ with initial data $\left(\vartheta_{0}, \eta_{0}\right)$ given at time $\tau \leq t$ as

$$
\left(\vartheta(t), \eta^{t}\right)=U_{f}^{\omega}(t, \tau)\left(\vartheta_{0}, \eta_{0}\right) .
$$

The two-parameter family $U_{f}^{\omega}(t, \tau)$ fulfils the following relations:

1. $U_{f}^{\omega}(t, \tau): \mathcal{H} \rightarrow \mathcal{H}$ for any $t \geq \tau, \tau \in \mathbb{R}$.

2. $U_{f}^{\omega}(\tau, \tau)$ is the identity map on $\mathcal{H}$ for any $\tau \in \mathbb{R}$.

3. $U_{f}^{\omega}(t, s) U_{f}^{\omega}(s, \tau)=U_{f}^{\omega}(t, \tau)$ for any $t \geq s \geq \tau, \tau \in \mathbb{R}$. 
4. $U_{f}^{\omega}(t, \tau) z \rightarrow z$ as $t \downarrow \tau$ for any $z \in \mathcal{H}, \tau \in \mathbb{R}$.

5. $U_{f}^{\omega}(t, \tau) \in C^{0}(\mathcal{H}, \mathcal{H})$ for any $\tau \in \mathbb{R}, t \geq \tau$.

The dependence of the process on $\omega$ is understood. It is convenient however to highlight the dependence on the external force $f$. This because we have in mind to consider, rather than a single process, a family of processes $\left\{U_{f}^{\omega}(t, \tau): f \in \mathcal{F}\right\}$, where $\mathcal{F}$, usually called the symbol space, is a suitable function space. The particular choice of $\mathcal{F}$ depends on which results one wants to prove (see Theorem 4.1 and the beginning of Section 5).

For further reference, it is useful to write problem $\mathbf{P}_{\omega}$ in a more compact form. Hence, setting

$$
z_{0}=\left(\vartheta_{0}, \eta_{0}\right) \quad \text { and } \quad z(t)=\left(\vartheta(t), \eta^{t}\right)
$$

we have

$$
\begin{aligned}
\frac{d}{d t} z(t) & =L_{\omega} z(t)+N(\cdot, z(t))+F(t) \\
z(\tau) & =z_{0}
\end{aligned}
$$

where the linear operator $L_{\omega}$ is defined as

$$
L_{\omega}=\left(\begin{array}{cc}
\omega \Delta & \int_{0}^{\infty} \mu(s) \Delta \cdot d s \\
\mathbb{I} & -\partial_{s}
\end{array}\right)
$$

with domain

$$
\mathcal{D}\left(L_{\omega}\right)=\left\{\begin{array}{l|l}
z \in \mathcal{H} & \begin{array}{l}
\vartheta \in H_{0}^{1} \\
\omega \Delta \vartheta+\int_{0}^{\infty} \mu(s) \Delta \eta(s) d s \in L^{2} \\
\partial_{s} \eta(s) \in L_{\mu}^{2}\left(\mathbb{R}^{+}, H_{0}^{1}\right) \\
\eta(0)=0
\end{array}
\end{array}\right\}
$$

and the non-linearity $N$ and the source term $F$ are given by

$$
N(\cdot, z)=(g(\cdot, \vartheta), 0) \quad \text { and } \quad F(t)=(f(t), 0)
$$

The energy associated to (3.9) at time $t$ is given by

$$
\mathcal{E}(t)=\frac{1}{2}\left\|U_{f}^{\omega}(t, \tau) z_{0}\right\|_{\mathcal{H}}^{2}=\frac{1}{2}\left(\|u(t)\|^{2}+\int_{0}^{\infty} \mu(s)\left\|\nabla \eta^{t}(s)\right\|^{2} d s\right)
$$




\section{Uniform absorbing sets}

The main result of this section is the following uniform in time energy estimate.

Theorem 4.1. Assume (h1) - (h5) and (g1) - (g3). Let $\mathcal{F} \subset \mathcal{T}\left(L^{2}\right)$ be a bounded set. Then there exist positive constants $C$, $\varepsilon$, and $\Lambda=\Lambda(\mathcal{F})$, all independent of $\omega$, such that the relation

$$
\mathcal{E}(t) \leq C e^{-\varepsilon(t-\tau)} \mathcal{E}(\tau)+\Lambda
$$

holds for every $t \geq \tau$, every $\tau \in \mathbb{R}$, and every $f \in \mathcal{F}$. In particular, if $g \equiv 0$ and $\mathcal{F}$ reduces to the null function (that is, the linear homogeneous case), then $\Lambda=0$.

Proof. Let $f \in \mathcal{F}$ be fixed. We perform formal estimates, that can be made rigorous in an approximation scheme. Notice first that, by the continuity of $g$ and (g3), for every $\nu>0$ there exists $c_{3}=c_{3}(\nu)$ such that

$$
r g(x, r) \leq \nu r^{2}+c_{3} \quad(x \in \Omega, r \in \mathbb{R}) .
$$

Therefore, the product in $\mathcal{H}$ of (3.9) and $z$ entail

$$
\begin{aligned}
\frac{d}{d t} \mathcal{E} & =-\omega\|\nabla \vartheta\|^{2}+\langle g(\cdot, \vartheta), \vartheta\rangle+\langle f, \vartheta\rangle-\int_{0}^{\infty} \mu(s)\left\langle\nabla \eta_{s}(s), \nabla \eta(s)\right\rangle d s \\
& \leq-\omega\|\nabla \vartheta\|^{2}+\nu\|\vartheta\|^{2}+c_{3}|\Omega|+\|f\|\|\vartheta\|-\int_{0}^{\infty} \mu(s)\left\langle\nabla \eta_{s}(s), \nabla \eta(s)\right\rangle d s
\end{aligned}
$$

Notice that $c_{3}$ vanishes when $g \equiv 0$. Reasoning as in the proof of Theorem 3.1, integration by parts and (h4) supply

$$
\begin{aligned}
-\int_{0}^{\infty} \mu(s)\left\langle\nabla \eta_{s}(s), \nabla \eta(s)\right\rangle d s & =\frac{1}{2} \int_{0}^{\infty} \mu^{\prime}(s)\|\nabla \eta(s)\|^{2} d s \\
& \leq-\frac{\delta}{2} \int_{0}^{\infty} \mu(s)\|\nabla \eta(s)\|^{2} d s
\end{aligned}
$$

Take now the product in $L^{2}$ of the first equation of system (3.9) and $-\eta(s)$, and integrate over $\mathbb{R}^{+}$in $\mu(s) d s$. This gives

$$
\begin{aligned}
-\int_{0}^{\infty} \mu(s)\left\langle\vartheta_{t}, \eta(s)\right\rangle d s= & \omega \int_{0}^{\infty} \mu(s)\langle\nabla \vartheta, \nabla \eta(s)\rangle d s+\left\|\int_{0}^{\infty} \mu(s) \nabla \eta(s) d s\right\|^{2} \\
& -\int_{0}^{\infty} \mu(s)\langle g(\cdot, \vartheta), \eta(s)\rangle d s-\int_{0}^{\infty} \mu(s)\langle f, \eta(s)\rangle d s .
\end{aligned}
$$

By means of Hölder and Young inequalities, (h3), (g1), (2.1), we estimate the terms of the right-hand side of (4.4) as follows:

$$
\begin{gathered}
\omega \int_{0}^{\infty} \mu(s)\langle\nabla \vartheta, \nabla \eta(s)\rangle d s \leq \omega\|\nabla \vartheta\|^{2}+\frac{\omega k_{0}}{4} \int_{0}^{\infty} \mu(s)\|\nabla \eta(s)\|^{2} d s \\
\left\|\int_{0}^{\infty} \mu(s) \nabla \eta(s) d s\right\|^{2} \leq\left(\int_{0}^{\infty} \mu(s)\|\nabla \eta(s)\| d s\right)^{2} \leq k_{0} \int_{0}^{\infty} \mu(s)\|\nabla \eta(s)\|^{2} d s
\end{gathered}
$$




$$
\begin{aligned}
& -\int_{0}^{\infty} \mu(s)\langle g(\cdot, \vartheta), \eta(s)\rangle d s \\
& \quad \leq \frac{c_{1} k_{0}|\Omega|}{2}+\frac{k_{0}}{4}\|\vartheta\|^{2}+c_{1} \lambda_{0}\left(c_{1}+\frac{1}{2}\right) \int_{0}^{\infty} \mu(s)\|\nabla \eta(s)\|^{2} d s \\
& -\int_{0}^{\infty} \mu(s)\langle f, \eta(s)\rangle d s \leq k_{0}^{\frac{1}{2}} \lambda_{0}^{\frac{1}{2}}\|f\|\left(\int_{0}^{\infty} \mu(s)\|\nabla \eta(s)\|^{2} d s\right)^{\frac{1}{2}} .
\end{aligned}
$$

Introducing the functional

$$
\mathcal{L}(t)=-\int_{0}^{\infty} \mu(s)\langle\vartheta, \eta(s)\rangle d s
$$

and exploiting the second equation of system (3.9), we can transform the left-hand side of (4.4) into

$$
-\int_{0}^{\infty} \mu(s)\left\langle\vartheta_{t}, \eta(s)\right\rangle d s=\frac{d}{d t} \mathcal{L}+k_{0}\|\vartheta\|^{2}-\int_{0}^{\infty} \mu(s)\left\langle\vartheta, \eta_{s}(s)\right\rangle d s
$$

Finally, the Hölder inequality, (h2) - (h3), (h5), and (2.1), entail

$$
\begin{aligned}
& \int_{0}^{\infty} \mu(s)\left\langle\vartheta, \eta_{s}(s)\right\rangle d s \\
& =-\int_{0}^{\infty} \mu^{\prime}(s)\langle\vartheta, \eta(s)\rangle d s \\
& \leq \lambda_{0}^{\frac{1}{2}}\|\vartheta\|\left(\frac{1}{\mu^{\frac{1}{2}}\left(s_{0}\right)} \int_{0}^{s_{0}}\left|\mu^{\prime}(s)\right| \mu^{\frac{1}{2}}(s)\|\nabla \eta(s)\| d s+M \int_{s_{0}}^{\infty} \mu(s)\|\nabla \eta(s)\| d s\right) \\
& \leq \lambda_{0}^{\frac{1}{2}}\|\vartheta\|\left\{\frac{1}{\mu^{\frac{1}{2}}\left(s_{0}\right)}\left(\int_{0}^{s_{0}}\left|\mu^{\prime}(s)\right|^{2} d s\right)^{\frac{1}{2}}\left(\int_{0}^{s_{0}} \mu(s)\|\nabla \eta(s)\|^{2} d s\right)^{\frac{1}{2}}\right. \\
& \left.\quad+M k_{0}^{\frac{1}{2}}\left(\int_{s_{0}}^{\infty} \mu(s)\|\nabla \eta(s)\|^{2} d s\right)^{\frac{1}{2}}\right\} \\
& \leq \frac{k_{0}}{4}\|\vartheta\|^{2}+c_{4} \int_{0}^{\infty} \mu(s)\|\nabla \eta(s)\|^{2} d s
\end{aligned}
$$

where

$$
c_{4}=\lambda_{0} \max \left\{\frac{1}{k_{0} \mu\left(s_{0}\right)} \int_{0}^{s_{0}}\left|\mu^{\prime}(s)\right|^{2} d s, M^{2}\right\} .
$$

Collecting (4.4) - (4.9), and denoting for simplicity

$$
\begin{aligned}
& c_{5}=\frac{\omega k_{0}}{4}+k_{0}+c_{1} \lambda_{0}\left(c_{1}+\frac{1}{2}\right)+c_{4} \\
& c_{6}=k_{0}^{\frac{1}{2}} \lambda_{0}^{\frac{1}{2}} \\
& c_{7}=\frac{c_{1} k_{0}|\Omega|}{2}
\end{aligned}
$$


we get the inequality

$$
\begin{aligned}
& \frac{d}{d t} \mathcal{L}+\frac{k_{0}}{2}\|\vartheta\|^{2} \\
& \quad \leq \omega\|\nabla \vartheta\|^{2}+c_{5} \int_{0}^{\infty} \mu(s)\|\nabla \eta(s)\|^{2} d s+c_{6}\|f\|\left(\int_{0}^{\infty} \mu(s)\|\nabla \eta(s)\|^{2} d s\right)^{\frac{1}{2}}+c_{7}
\end{aligned}
$$

Notice that $c_{7}=0$ when $g \equiv 0$.

For $N>0$, set

$$
\Phi(t)=N \mathcal{E}(t)+\mathcal{L}(t) .
$$

Then fix $N>2$ big enough such that

$$
\frac{1}{c_{8}} \mathcal{E}(t) \leq \Phi(t) \leq c_{8} \mathcal{E}(t)
$$

for some $c_{8}>1$, and

$$
\frac{N \delta}{2}-c_{5} \geq \frac{k_{0}}{4} .
$$

Addition of (4.2) and $N$-times (4.11), and (4.3) and (4.13), lead to

$$
\begin{aligned}
\frac{d}{d t} \Phi & +(N-1) \omega\|\nabla \vartheta\|^{2}+\left(\frac{k_{0}}{2}-N \nu\right)\|\vartheta\|^{2}+\frac{k_{0}}{4} \int_{0}^{\infty} \mu(s)\|\nabla \eta(s)\|^{2} d s \\
& \leq N\|f\|\|\vartheta\|+c_{6}\|f\|\left(\int_{0}^{\infty} \mu(s)\|\nabla \eta(s)\|^{2} d s\right)^{\frac{1}{2}}+N c_{3}|\Omega|+c_{7} .
\end{aligned}
$$

At this point, we fix the value of $\nu>0$ small enough such that

$$
\frac{k_{0}}{2}-N \nu \geq \frac{k_{0}}{4}
$$

Hence, setting

$$
\varepsilon=\frac{k_{0}}{2 c_{8}}, \quad c_{9}=c_{6} \sqrt{2 c_{8}}+N \sqrt{c_{8}}, \quad c_{10}=N c_{3}|\Omega|+c_{7}
$$

inequalities (4.14) - (4.15) yield

$$
\frac{d}{d t} \Phi+\varepsilon \Phi \leq c_{9}\|f\| \Phi^{\frac{1}{2}}+c_{10}
$$

where $c_{10}=0$ when $g \equiv 0$. Exploiting a Gronwall-type Lemma (cf. [3, 21]), we get from (4.16) the inequality

$$
\Phi(t) \leq 2 \Phi(\tau) e^{-\varepsilon(t-\tau)}+\frac{2 c_{10}}{\varepsilon}+\frac{c_{9}^{2} e^{\varepsilon}}{\left(1-e^{-\frac{\varepsilon}{2}}\right)^{2}}\|f\|_{\mathcal{T}\left(L^{2}\right)}^{2} .
$$

The thesis then follows at once from (4.12) 
It is worth noting that, in particular, here we provided a direct proof of the exponential decay of the strongly continuous semigroup associated to the linear homogeneous case. The reader should confront this result with [15].

Corollary 4.2. Let the hypotheses of Theorem 4.1 be fulfilled. Then there exists an invariant, bounded, absorbing set $\mathcal{B}_{0}$ for the family $\left\{U_{f}(t, \tau), f \in \mathcal{F}\right\}$, which is uniform as $\omega \geq 0$ and $f \in \mathcal{F}$. Moreover, $\mathcal{B}_{0}$ is connected whenever $\mathcal{F}$ is connected.

Proof. Just define

$$
\mathcal{B}_{0}=\bigcup_{\omega \geq 0} \bigcup_{f \in \mathcal{F}} \bigcup_{\tau \in \mathbb{R}} \bigcup_{t \geq \tau} U_{f}^{\omega}(t, \tau) \mathcal{B}
$$

where $\mathcal{B}$ is the ball of $\mathcal{H}$ of radius $2 \sqrt{\Lambda}$ centered at zero

Another straightforward consequence of Theorem 4.1 is the following result, which will be needed later.

Corollary 4.3. Assume (h1) - (h5) and (g1) - (g3). Let $\mathcal{F} \subset \mathcal{T}\left(L^{2}\right)$ be a bounded set. Then there exist a positive constant $C$ depending on $\mathcal{F}$, but independent of $\omega$, such that the relation

$$
\omega \int_{\tau}^{t}\|\nabla \vartheta\|^{2} \leq C(\mathcal{E}(0)+t-\tau)
$$

holds for every $t \geq \tau$, every $\tau \in \mathbb{R}$, and every $f \in \mathcal{F}$.

\section{Existence of a uniform attractor}

In the sequel, let (h1) - (h5) and (g1) - (g4) hold. We assume

$$
h \text { is translation compact in } L_{\text {loc }}^{1}\left(\mathbb{R}, H_{0}^{1}\right) \text {. }
$$

Then we take $\mathcal{F}=\mathrm{H}(h)$, and we study the asymptotic behavior of the family of processes $\left\{U_{f}^{\omega}(t, \tau), f \in \mathrm{H}(h)\right\}$, for $\omega \geq 0$.

Recall that a set $\mathcal{A}_{\omega} \subset \mathcal{H}$ is said to be the uniform attractor for the family of processes $\left\{U_{f}^{\omega}(t, \tau), f \in \mathrm{H}(h)\right\}$ if it is at the same time a uniformly (as $f \in \mathrm{H}(h)$ ) attracting set, and it is contained in every closed uniformly attracting set. The reader is referred to the classical books $[2,18,20,23]$ for an overview of the relevant concepts and results used in this section.

When $\omega>0$, as shown in [13], the family $\left\{U_{f}^{\omega}(t, \tau), f \in \mathrm{H}(h)\right\}$ possesses a uniform attractor, even assuming weaker conditions on $g$, and for $h$ translation compact in $L_{\text {loc }}^{1}\left(\mathbb{R}, L^{2}\right)$. Indeed, the dissipation contained in $\Delta \vartheta$ plays a significant role. In this work, however, we are mainly interested to the case $\omega=0$, and to the continuity properties that we can obtain as $\omega \rightarrow 0$. This is why we will look for results which are independent of $\omega$. Clearly, in the estimates that follow, our assumptions on $g$ and $h$ will not be optimal for a fixed $\omega>0$, but they probably are as $\omega \rightarrow 0$ (and, in particular, for $\omega=0)$. 
Let $\mathcal{B}_{0}$ be the invariant, bounded, connected, uniform absorbing set of $U_{f}^{\omega}(t, \tau)$, given by Corollary 4.2. For any $z_{0} \in \mathcal{B}_{0}$, we write the solution $z$ to $(3.9)$ as $z=z_{c}+z_{d}$, where $z_{c}$ and $z_{d}$ are the solutions to the problems

$$
\left.\begin{array}{rl}
\frac{d}{d t} z_{c} & =L_{\omega} z_{c}+N\left(\cdot, z_{c}\right)+F \\
z_{c}(\tau) & =0
\end{array}\right\}
$$

and

$$
\left.\begin{array}{rl}
\frac{d}{d t} z_{d} & =L_{\omega} z_{d}+N(\cdot, z)-N\left(\cdot, z_{c}\right) \\
z_{d}(\tau) & =z_{0}
\end{array}\right\},
$$

respectively. It is immediate to see (cf. Theorem 3.2) that problems (5.2) and (5.3) admit unique solutions belonging to the space $C^{0}([\tau, \infty), \mathcal{H})$. Introducing the product Hilbert space

$$
\mathcal{V}=H_{0}^{1} \times L_{\mu}^{2}\left(\mathbb{R}^{+}, H^{2} \cap H_{0}^{1}\right)
$$

we have

Lemma 5.1. There exists a constant $K>0$ such that the inequality

$$
\left\|z_{c}(t)\right\|_{\mathcal{V}} \leq K
$$

holds for every $\tau \in \mathbb{R}, t \geq \tau, \omega \geq 0$, and $z_{0} \in \mathcal{B}_{0}$.

Proof. We repeat the proof of Theorem 4.1, except we now take inner products in $H_{0}^{1}$ and $H^{2} \cap H_{0}^{1}$ rather then in $L^{2}$ and $H_{0}^{1}$, respectively. This is possible since, on account of $(\mathrm{g} 4), g\left(x, \vartheta_{c}(x, t)\right) \in H_{0}^{1}$. Thus we define

$$
\mathcal{E}_{c}(t)=\frac{1}{2}\left(\left\|\nabla u_{c}(t)\right\|^{2}+\int_{0}^{\infty} \mu(s)\left\|\Delta \eta_{c}^{t}(s)\right\|^{2} d s\right)
$$

and

$$
\mathcal{L}_{c}(t)=-\int_{0}^{\infty} \mu(s)\left\langle\nabla \vartheta_{c}, \nabla \eta_{c}(s)\right\rangle d s
$$

Notice that Theorem 4.1 applies to $\mathcal{E}_{c}$ as well. In particular, this means that $\left\|\vartheta_{c}\right\|$ is uniformly bounded. Hence, in force of $(\mathrm{g} 4)$ and the Young inequality,

$$
\left\langle\nabla g\left(\cdot, \vartheta_{c}\right), \nabla \vartheta_{c}\right\rangle=\left\langle D_{x} g\left(\cdot, \vartheta_{c}\right), \nabla \vartheta_{c}\right\rangle+\left\langle g^{\prime}\left(\cdot, \vartheta_{c}\right) \nabla \vartheta_{c}, \nabla \vartheta_{c}\right\rangle \leq \nu\left\|\nabla \vartheta_{c}\right\|^{2}+c_{11}
$$

for every $\nu>0$ and some $c_{11}=c_{11}(\nu)$. Therefore, the analog of $(4.2)-(4.3)$ reads

$$
\begin{aligned}
\frac{d}{d t} \mathcal{E}_{c} & +\frac{1}{2} \int_{0}^{\infty} \mu(s)\left\|\Delta \eta_{c}(s)\right\|^{2} d s \\
& \leq-\omega\left\|\Delta \vartheta_{c}\right\|^{2}+\left\langle\nabla g\left(\cdot, \vartheta_{c}\right), \nabla \vartheta_{c}\right\rangle+\left\langle\nabla f, \nabla \vartheta_{c}\right\rangle \\
& \leq-\omega\left\|\Delta \vartheta_{c}\right\|^{2}+\nu\left\|\nabla \vartheta_{c}\right\|^{2}+c_{11}+\|\nabla f\|\left\|\nabla \vartheta_{c}\right\|
\end{aligned}
$$


Estimates (4.4) - (4.6) and (4.8) - (4.10) hold for $\left(\vartheta_{c}, \eta_{c}\right)$, provided we replace norms and inner products properly. The only difference is in (4.7). Here, using (2.1), (h1), and (g1), we have

$$
\begin{aligned}
-\int_{0}^{\infty} \mu(s)\left\langle\nabla g\left(\cdot, \vartheta_{c}\right), \nabla \eta_{c}(s)\right\rangle d s & =\int_{0}^{\infty} \mu(s)\left\langle g\left(\cdot, \vartheta_{c}\right), \Delta \eta_{c}(s)\right\rangle d s \\
& \leq c_{12}+\frac{k_{0}}{4}\left\|\vartheta_{c}\right\|^{2}+c_{13} \int_{0}^{\infty} \mu(s)\left\|\nabla \eta_{c}(s)\right\|^{2} d s
\end{aligned}
$$

for some $c_{12}, c_{13}>0$. Reasoning as in the proof of Theorem 4.1 (that is, defining $\Phi_{c}=N \mathcal{E}_{c}+\mathcal{L}_{c}$ ), exploiting (5.4) - (5.5), and recalling that $z_{c}(\tau)=0$, which implies $\Phi_{c}(\tau)=0$, we get the thesis. Clearly, the constant $K$ of the lemma shall depend on $\|h\|_{\mathcal{T}\left(H_{0}^{1}\right)}$

At this level, we cannot conclude that the orbits $z_{c}(t)$ lie in a relatively compact subset of $\mathcal{H}$. Indeed, even if we have boundedness in $\mathcal{V}$, the embedding $\mathcal{V} \hookrightarrow \mathcal{H}$ lacks of compactness. To overcome this obstacle, we shall treat separately the second component $\eta_{c}$ of $z_{c}$, along the direction suggested by [22].

Lemma 5.2. For every $\omega \geq 0$, the set

$$
\mathcal{E}=\bigcup_{f \in \mathrm{H}(h)} \bigcup_{z_{0} \in \mathcal{B}_{0}} \bigcup_{\tau \in \mathbb{R}} \bigcup_{t \geq \tau} \eta_{c}^{t}
$$

is relatively compact in $L_{\mu}^{2}\left(\mathbb{R}^{+}, H_{0}^{1}\right)$, and bounded in $H_{\mu}^{1}\left(\mathbb{R}^{+}, H_{0}^{1}\right) \cap L_{\mu}^{2}\left(\mathbb{R}^{+}, H^{2} \cap H_{0}^{1}\right)$, uniformly as $\omega \geq 0$.

Proof. Since $\eta_{c}^{t}$ has the explicit form (see [13])

$$
\eta_{c}^{t}(s)= \begin{cases}\int_{0}^{s} \vartheta_{c}(t-y) d y & \text { for } 0<s \leq t-\tau \\ \int_{0}^{t-\tau} \vartheta_{c}(t-y) d y & \text { for } s>t-\tau\end{cases}
$$

and consequently

$$
\partial_{s} \eta_{c}^{t}(s)= \begin{cases}\vartheta_{c}(t-s) & \text { for } 0<s \leq t-\tau \\ 0 & \text { for } s>t-\tau\end{cases}
$$

it is easy to conclude from Lemma 5.1 and (h4) that

$$
\mathcal{E} \text { is bounded in } H_{\mu}^{1}\left(\mathbb{R}^{+}, H_{0}^{1}\right) \cap L_{\mu}^{2}\left(\mathbb{R}^{+}, H^{2} \cap H_{0}^{1}\right)
$$

and

$$
\sup _{\eta \in \mathcal{E}} \int_{0}^{\infty}\|\nabla \eta(s)\|^{2} d s \in L_{\mu}^{1}\left(\mathbb{R}^{+}\right)
$$

(the above bounds being independent of $\omega \geq 0$ ). The result then follows from [22: Lemma 5.5] (see also $[11,13])$ 
Corollary 5.3. There exists a relatively compact set $\mathcal{K} \subset \mathcal{H}$, independent of $\omega \geq 0$, such that

$$
\bigcup_{f \in \mathrm{H}(h)} \bigcup_{z_{0} \in \mathcal{B}_{0}} \bigcup_{\tau \in \mathbb{R}} \bigcup_{t \geq \tau} z_{c}(t) \subset \mathcal{K}
$$

Moreover, $\mathcal{K}$ is bounded in $\mathcal{V}$, and its second component is also bounded in $H_{\mu}^{1}\left(\mathbb{R}^{+}, H_{0}^{1}\right)$.

Proof. Just denote $\mathcal{K}=\mathcal{B}_{K} \times \mathcal{E}$, where $\mathcal{B}_{K}$ is the closed ball of $H_{0}^{1}$ of radius $K(\rho)$ centered at zero

Finally, we turn our attention to system (5.3).

Lemma 5.4. There exist positive constants $C$ and $\varepsilon$ independent of $\omega \geq 0$ such that the relation

$$
\left\|z_{d}(t)\right\|_{\mathcal{H}} \leq C e^{-\varepsilon(t-\tau)}
$$

holds for every $\tau \in \mathbb{R}, t \geq \tau, \omega \geq 0$ and $z_{0} \in \mathcal{B}_{0}$.

Proof. Again, we mimic the proof of Theorem 4.2. Just notice that, with reference to (4.2) and (4.7),

$$
\left\langle g(\cdot, \vartheta)-g\left(\cdot, \vartheta_{c}\right), \vartheta_{d}\right\rangle \leq 0
$$

thanks to $(\mathrm{g} 4)$, and

$$
\begin{aligned}
-\int_{0}^{\infty} & \mu(s)\left\langle g(\cdot, \vartheta)-g\left(\cdot, \vartheta_{c}\right), \eta_{d}(s)\right\rangle d s \\
\leq & c_{2} \int_{0}^{\infty} \mu(s)\left\|\vartheta_{d}\right\|\left\|\eta_{d}(s)\right\| d s \\
\quad \leq & \frac{k_{0}}{4}\left\|\vartheta_{d}\right\|^{2}+c_{2}^{2} \lambda_{0} \int_{0}^{\infty} \mu(s)\left\|\nabla \eta_{d}(s)\right\|^{2} d s
\end{aligned}
$$

thanks to (g2), (2.1), and the Young inequality. Further details are left to the reader

Remark 5.5. It should be noted that the above results still hold if we weaken condition $(\mathrm{g} 4)$, asking that $g^{\prime}(\cdot, r) \leq \nu$, for some small constant $\nu$, whose upper limit can be explicitly found with a more careful balance of the estimates appearing in Lemmas 5.1 and 5.4.

Collecting Corollary 5.3 and Lemma 5.4, it is apparent to check that the relatively compact set $\mathcal{K}$ has the uniform attraction property, that is, for any $\tau \in \mathbb{R}$, and any bounded set $\mathcal{B} \subset \mathcal{H}$,

$$
\lim _{t \rightarrow \infty}\left[\sup _{f \in \mathcal{F}} \delta_{\mathcal{H}}\left(U_{f}^{\omega}(t, \tau) \mathcal{B}, \mathcal{K}\right)\right]=0
$$

where $\delta_{\mathcal{H}}$ is the Hausdorff semidistance on $\mathcal{H}$, defined by

$$
\delta_{\mathcal{H}}\left(\mathcal{B}_{1}, \mathcal{B}_{2}\right)=\sup _{b_{1} \in \mathcal{B}_{1}} \operatorname{dist}_{\mathcal{H}}\left(b_{1}, \mathcal{B}_{2}\right)=\sup _{b_{1} \in \mathcal{B}_{1}} \inf _{b_{2} \in \mathcal{B}_{2}}\left\|b_{1}-b_{2}\right\|_{\mathcal{H}}
$$

Furthermore, from Theorem 3.2 we know that $U_{\bullet}(t, \tau)$ is continuous as a map $\mathcal{H} \times$ $\mathrm{H}(g) \rightarrow \mathcal{H}$.

Using the above continuity property, together with (5.6), we can apply standard results (see $[4,5,11,13])$ to get 
Theorem 5.6. Assume (h1) - (h5), (g1)-(g4) and (5.1) hold. Then, for every $\omega \geq 0$, the family of processes $\left\{U_{f}^{\omega}(t, \tau), f \in \mathrm{H}(h)\right\}$ associated with problem $\mathbf{P}_{\omega}$ possesses a compact and connected uniform attractor $\mathcal{A}_{\omega}$ given by

$$
\mathcal{A}_{\omega}=\left\{\begin{array}{l}
z(0) \text { such that } z(t) \text { is any bounded complete } \\
\text { trajectory of } U_{f}^{\omega}(t, \tau) \text { for some } f \in \mathrm{H}(h)
\end{array}\right\} .
$$

Recall that the uniform attractor is the smallest compact set of $\mathcal{H}$ which enjoys the uniform attraction property (see $[4,5,20]$ for more details). In particular, due to the minimality property of uniform attractors among uniform attracting sets, from Corollary 5.3 we also get

Corollary 5.7. The uniform attractor $\mathcal{A}_{\omega}$ is bounded in $\mathcal{V}$, and its second component is also bounded in $H_{\mu}^{1}\left(\mathbb{R}^{+}, H_{0}^{1}\right)$. The bounds are independent of $\omega \geq 0$.

Remark 5.8. When $h$ is quasi periodic, namely

$$
h(x, t)=\Psi(x, \kappa t)=\Psi\left(x, \kappa_{1} t, \ldots, \kappa_{m} t\right)
$$

where $\Psi(\cdot, \varpi) \in C^{1}\left(\mathbf{T}^{m}, H_{0}^{1}\right)$ is a $2 \pi$-periodic function of $\varpi$ on the $m$-dimensional torus $\mathbf{T}^{m}$, and $\kappa_{1}, \ldots, \kappa_{m}$ are rationally independent numbers, then, arguing as in [13] (cf. also [16]), it is possible to show that, for every $\omega \geq 0$, the uniform attractor $\mathcal{A}_{\omega}$ of the family $\left\{U_{f}^{\omega}(t, \tau), f \in \mathrm{H}(h)\right\}$ has finite fractal dimension. The reader is referred to $[8$, $23]$ for more details on the subject.

\section{The Hyperbolic limit}

In this last section we examine the behavior of the longtime solutions to problem $\mathbf{P}_{\omega}$ in the hyperbolic limit $\omega \rightarrow 0$. The result we obtain will show that, in the limit, the dynamics of the parabolic problem tend to coincide with the dynamics of the hyperbolic problem. The argument an be made precise in terms of Hausdorff semidistance of the respective attractors.

Just for sake of simplicity, we take $f \in H_{0}^{1}$ constant in time. Then, as remarked in the introduction,

$$
S_{\omega}(t)=U_{f}^{\omega}(t, 0)
$$

turns out to be a strongly continuous semigroup. Moreover, throughout this section, we shall assume that conditions (h1) - (h5) and (g1) - (g4) hold.

In fact, the following argument can be immediately adapted to the non-autonomous setting, provided we work with the semigroup $\Sigma_{\omega}(t)$, acting on the complete metric space $\mathcal{H} \times \mathrm{H}(h)$, defined by (cf. $[4-6])$

$$
\Sigma_{\omega}(t)(z, f)=\left(U_{f}^{\omega}(t, 0) z, f^{t}\right) .
$$

We set

$$
\mathcal{A}=\bigcup_{\omega \geq 0} \mathcal{A}_{\omega} .
$$

The invariance relation $S_{\omega}(t) \mathcal{A}_{\omega}=\mathcal{A}_{\omega}$, which holds for every $\omega \geq 0$, and Corollary 5.7 imply 
Lemma 6.1. The set $\mathcal{A}$ is relatively compact, is bounded in $\mathcal{V}$, and its third component is also bounded in $H_{\mu}^{1}\left(\mathbb{R}^{+}, H_{0}^{1}\right)$.

Concerning the continuity properties of $S_{\omega}(t)$ as a function of $\omega$, we have

Lemma 6.2. For every $\omega_{0}>0$ there exists a constant $C=C\left(\omega_{0}\right)$ such that

$$
\left\|S_{\omega_{1}}(t) z_{0}-S_{\omega_{2}}(t) z_{0}\right\|_{\mathcal{H}} \leq C \sqrt{t}
$$

for every $\omega_{1}, \omega_{2} \leq \omega_{0}$ and every $z_{0} \in \mathcal{A}$.

Proof. Let $z_{j}$, for $j=1,2$, be the solutions to problem $\mathbf{P}_{\omega_{j}}$, with initial data $z_{j}(0)=z_{0}$. Denoting $z=z_{1}-z_{2}$, we obtain the system

$$
\begin{aligned}
& \vartheta_{t}=\omega_{1} \Delta \vartheta_{1}-\omega_{2} \Delta \vartheta_{2}+\int_{0}^{\infty} \mu(s) \Delta \eta(s) d s+g\left(\cdot, \vartheta_{1}\right)-g\left(\cdot, \vartheta_{2}\right) \\
& \eta_{t}+\eta_{s}=\vartheta
\end{aligned}
$$

The result follows immediately multiplying the above system by $(\vartheta, \eta)$ in $\mathcal{H}$, using the fact that $z_{j}(t) \in \mathcal{A}$ for every $t \geq 0$, and exploiting (g1) and Lemma 6.1

At this point, we fix $\omega_{0}>0$, and we introduce the set $\mathcal{G}$ as

$$
\mathcal{G}=\left\{z_{\omega} \in C^{0}([0, \infty), \mathcal{H}): z_{\omega}(t)=S_{\omega}(t) z_{\omega 0}, \text { with } z_{\omega 0} \in \mathcal{A}_{\omega}, \text { for } \omega \leq \omega_{0}\right\}
$$

We have

Lemma 6.3. $\mathcal{G}$ is an equicontinuous family at zero.

Proof. We re-cast the argument used in [16], of which here we limit ourselves to give the essential lines. First, one has to exploit the strong continuity at zero of $S_{\omega}(t)$, for every fixed $\omega$. Then it is possible to find a finite cover of $\mathcal{A}$ (due to Lemma 6.1) of balls af radius $\varepsilon$, and the desired result follows applying the Minkowski inequality to the difference $\left\|S_{\omega}(t) z_{\omega}-z_{\omega}\right\|_{\mathcal{H}}$, on account of (3.2) and Lemma 6.2

The main result of this section is the following.

Theorem 6.4. The attractor $\mathcal{A}_{0}$ of $S_{0}(t)$ is upper semicontinuous at zero with respect to the sets $\left\{\mathcal{A}_{\omega}, \omega>0\right\}$, that is

$$
\lim _{\omega \rightarrow 0} \delta_{\mathcal{H}}\left(\mathcal{A}_{\omega}, \mathcal{A}_{0}\right)=0
$$

Proof. Along the lines of [19], by contradiction, assume that there exists a constant $\varrho>0$, a sequence of positive numbers $\omega_{n} \rightarrow 0$, and a corresponding sequence $z_{n 0}$ of $\mathcal{A}_{\omega_{n}}$ such that

$$
\operatorname{dist}_{\mathcal{H}}\left(z_{n 0}, \mathcal{A}_{0}\right) \geq \varrho
$$

We may suppose $\omega_{n} \leq \omega_{0}$ for every $n$. Let $z_{n}(t)=S_{\omega_{n}}(t) z_{n 0}$. Using the invariance of the attractor, it is possible to extend in a natural way $z_{n}(t)$ for all times. Just set, for $t<0, z_{n}(t)=z$, where $S_{\omega_{n}}(-t) z=z_{n 0}$. By Lemma 6.2, $\cup_{t \in \mathbb{R}} \cup_{n} z_{n}(t)$ is a relatively compact set in $\mathcal{H}$, and by Lemma 6.3 , the family of mappings $z_{n}$ is equicontinuous 
from $\mathbb{R}$ to $\mathcal{H}$. Indeed, the continuity at a point $t_{0} \neq 0$ is esily obtained exploiting the semigroup properties. Then Ascoli's theorem and a classical diagonalization method (cf. $[16,19])$ entails the existence of $\bar{z} \in C^{0}(\mathbb{R}, \mathcal{H})$ such that (up to a subsequence)

$$
z_{n} \longrightarrow \bar{z} \quad \text { in } C^{0}([-N, N], \mathcal{H})
$$

for every $N>0$. In particular, $z_{n 0} \longrightarrow \bar{z}(0)$ in $\mathcal{H}$. Moreover, $\sup _{t \in \mathbb{R}}\|\bar{z}(t)\|_{\mathcal{H}}<\infty$. Exploiting the above convergences, it is easy to show that $\bar{z}$ fulfils Definition 3.1 for $\omega=0$. We just dwell on the convergence of the term $-\omega_{n}\left\langle\nabla \vartheta_{n}, \nabla v\right\rangle$. By means of Corollary 4.3 , for every $t>\tau$ we have

$$
\omega_{n}\left|\int_{\tau}^{t}\left\langle\nabla \vartheta_{n}, \nabla v\right\rangle\right| \leq \sqrt{\omega_{n}}(t-\tau)^{\frac{1}{2}}\|\nabla v\|\left(\omega_{n} \int_{\tau}^{t}\left\|\nabla \vartheta_{n}\right\|^{2}\right)^{\frac{1}{2}} \longrightarrow 0 .
$$

Therefore $\bar{z}(t)$ is a bounded complete trajectory of $S_{0}(t)$, that is, $\bar{z}(t)$ solves problem $\mathbf{P}_{0}$ for all times and it is bounded in $\mathcal{H}$ uniformly in $t$. From the characterization of $\mathcal{A}_{0}$ (Theorem 5.6), we conclude that $\bar{z}(0) \in \mathcal{A}_{0}$. This implies that $\operatorname{dist}_{\mathcal{H}}\left(z_{n 0}, \mathcal{A}_{0}\right) \longrightarrow 0$ leading to a contradiction

\section{References}

[1] Amerio, L. and G. Prouse: Abstract Almost Periodic Functions and Functional Equations. New York: Van Nostrand 1971.

[2] Babin, A. V. and M. I. Vishik: Attractors of Evolution Equations. Amsterdam: NorthHolland 1992.

[3] Brézis, H.: Opérateurs maximaux monotones et semi-groupes de contractions dans les espaces de Hilbert (North-Holland Math. Stud.: Vol. 5). Amsterdam: North-Holland 1973.

[4] Chepyzhov, V.V. and M. I. Vishik: Nonautonomous evolution equations and their attractors. Russian J. Math. Phys. 1 (1993), 165 - 190.

[5] Chepyzhov, V.V. and M. I. Vishik: Attractors of non-autonomous dynamical systems and their dimension. J. Math. Pures Appl. 73 (1994), 279 - 333.

[6] Chepyzhov, V.V. and M. I. Vishik: Non-autonomous evolutionary equations with translation compact symbols and their attractor. C.R. Acad. Sci. Paris Sér. I Math. 321 (1995), $153-158$.

[7] Coleman, B. D. and M. E. Gurtin: Equipresence and constitutive equations for rigid heat conductors. Z. Angew. Math. Phys. 18 (1967), 199 - 208.

[8] Constantine, P., Foias, C. and R. Temam: Attractors representing turbulent flows. Mem. Amer. Math. Soc. 53 (1993), nr. 314.

[9] Dafermos, C. M.: Asymptotic stability in viscoelasticity. Arch. Rational Mech. Anal. 37 (1970), $297-308$.

[10] Giorgi, C., Grasselli, M. and V. Pata: Well-posedness and longtime behavior of the phasefield model with memory in a history space setting. Quart. Appl. Math. (to appear).

[11] Giorgi, C., Grasselli, M. and V. Pata: Uniform attractors for a phase-field model with memory and quadratic nonlinearity. Indiana U. Math. J. 48 (2000), 1395 - 1445. 
[12] Giorgi, C., Marzocchi, A. and V. Pata: Asymptotic behavior of a semilinear problem in heat conduction with memory. Nonlin. Diff. Equ. Appl. (NoDEA) 5 (1998), 333 - 354.

[13] Giorgi, C., Marzocchi, A. and V. Pata: Uniform attractors for a non-autonomous semilinear heat equation with memory. Quart. Appl. Math. 58 (2000), $661-683$.

[14] Giorgi, C., Muñoz Rivera, J. E. and V. Pata: Global attractors for a semilinear hyperbolic equation in viscoelasticity. J. Math. Anal. Appl. (to appear).

[15] Giorgi, C., Naso, M. G. and V. Pata: Exponential stability in linear heat conduction with memory: a semigroup approach. Comm. Appl. Anal. 5 (2001), $121-134$.

[16] Grasselli, M. and V. Pata: Upper semicontinuous attractors for a hyperbolic phase-field model with memory. Indiana U. Math. J. (to appear).

[17] Gurtin, M. E. and A. C. Pipkin: A general theory of heat conduction with finite wave speeds. Arch. Rational Mech. Anal. 31 (1968), 113 - 126.

[18] Hale, J.: Asymptotic behavior of dissipative systems. Providence (R.I., USA): Amer. Math. Soc. 1988.

[19] Hale, J. and G. Raugel: Upper semicontinuity of the attractor for a singularly perturbed hyperbolic equation. J. Diff. Equ. 73 (1988), 197 - 214.

[20] Haraux, A.: Systèmes dynamiques dissipatifs et applications (Coll. RMA: Vol. 17). Paris: Masson 1990.

[21] Pata, V., Prouse, G. and M. I. Vishik: Traveling waves of dissipative non-autonomous hyperbolic equations in a strip. Adv. Diff. Equ. 3 (1998), 249 - 270.

[22] Pata, V. and A. Zucchi: Attractors for a damped hyperbolic equations with linear memory. Adv. Math. Sci. Appl. (to appear).

[23] Temam, R.: Infinite-Dimensional Dynamical Systems in Mechanics and Physics. New York: Springer 1988.

Received 29.06.2000; in revised form 26.03.2001 